\title{
ON EXPLICIT SOLUTIONS OF NONLINEAR DYNAMIC SYSTEMS
}

\author{
Domine M.W. Leenaerts \\ Philips Research Laboratories \\ prof. Holstlaan 4, Eindhoven, Netherlands \\ e-mail: domine.leenaerts@philips.com
}

\begin{abstract}
A method is proposed to obtain explicit expressions for the large signal behavior in nonlinear dynamic circuits. The method is also an extension to the solution method for Linear Dynamic Complementary Problems.
\end{abstract}

\section{INTRODUCTION}

Recently, a technique was developed to obtain explicit formulas of the solutions of piecewise linear (PL) networks [1]. The basic idea behind the technique was simple. Any piecewise linear network can be reformulated into a Linear Complementary Problem (LCP). If this LCP is of class-P, the solutions of it can always be obtained

- in an algorithmic way and

- in an explicit form.

The class-P property is not restrictive in the sense that many electrical networks, including those with transistors, belong to this class if their nonlinear behavior is approximated by piecewise linear techniques [2]. Often these networks are referred to as class-P networks

Very recently, the technique was used to analyze class-P static networks in a symbolic manner [3]. The symbolic expressions for each current and voltage within the network cover the complete nonlinear piecewise linear approximated behavior within a single formula.

It is the goal of this paper to demonstrate that also for dynamic systems explicit formulas for the solutions of the networks variables can be obtained. This can be achieved by applying a linear multi step integration method to each dynamic element in the network. The integration method transforms each dynamic element into a small static linear network of which its components values are determined by the used time step size, the dynamic element value and the time history of integration. Then by applying the technique of obtaining the solutions of this static network results finally in
- explicit formulas for the solutions of the network at each time point

- the property that these solutions can be find in a symbolic manner

- the possibility to obtain explicit forms of the solutions for Linear Dynamic Complementary Problems (LDCP) [4]

We will start with some preliminaries on PL techniques in section 2. It will be shown that any $\mathrm{PL}$ model is related to the Linear Complementary Problem (LCP). Then, in section 3 a short outline of the proposed method will be given. To demonstrate the applicability of this result network examples will be provided and explained (section 4). In section 5 some words will be spent on the class- $P$ property. We will end up with some conclusions.

\section{PRELIMINARIES}

The piecewise linear (PL) network or mapping $f$ is assumed to be continuous. The function or mapping $f$ can be written in a certain PL model description and is inherently confronted with the Linear Complementary Problem (LCP) [2]. To make this more clear, the following model description is used

$$
\begin{gathered}
I y+A x+B u+f=0 \quad j=C x+I u+g \\
u \geq 0, j \geq 0, u^{T} \cdot j=0
\end{gathered}
$$

with which many network components can be modelled [5]. In (1), the first equation defines the linear relation between $y$ and $x$. The second equation defines in which region this linear relation holds. Changing from one region into another will effect the linear relation via state vector $u$, resulting in a new linear relation valid for the new entered region. Equation (2) reflects the complementary condition and is inherently nonlinear. Close related to $\mathrm{PL}$ modeling is the LCP. 
The LCP is defined as obtaining the solutions $u$ and $j$ of

$$
j=D u+q
$$

under restriction (2). As example consider the PL function of a nonlinear resistor in Fig. 1, described by

$$
I+\left(-\frac{1}{R_{2}}\right) V+\left(-\frac{1}{R_{2}}\right) u=0 \quad j=V+u
$$

The second equation of (4) together with (2) defines which linear segment is valid. When $V$ is nonnegative, $u$ has to be equal to zero. The current $I$ can then be obtained from the first equation of (4), $I=\frac{1}{R_{2}} V$. When $V$ is strictly negative, $j$ is equal to zero and $u=-V$. Using this result in the first equation results in the new linear relation $I=0$.
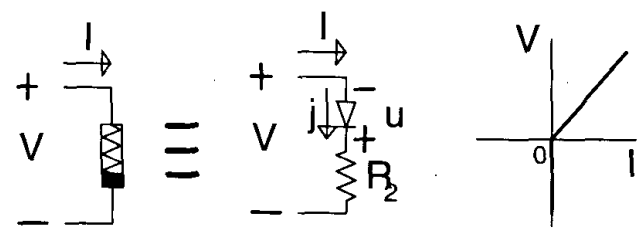

Fig.1. Nonlinear resistor with its PL equivalent network

Defining $q=V$, the second equation in (4) becomes $j=u+q$ and is therefore a special case of the LCP (3), i.e. $D=1$. LCP (3) yields an unique solution for any $q$ under the restriction that $D \in P$, where class- $P$ is strictly defined as in [2]. A definition for class- $P$ is that the principal minors of $D$ must be positive.

\section{THE METHOD}

In network simulators numerical integration methods are used to solve the linear time dependent equations of the network components [6]. Applying an integration method to a dynamic electrical network component results in a sequence of small static replacement circuits, where the component values in this circuit are determined by the used integration method, the step size and the history of integration.

Consider as example a linear capacitor $C$ and the well-known trapezoidal integration method [6]

$$
z_{n+1}=\frac{h}{2}\left(\dot{z}_{n+1}+\dot{z}_{n}\right)+z_{n}=P_{n}+\beta_{-1} \dot{z}_{n+1}
$$

(or the backward Euler formula $z_{n+1}=z_{n}+h \dot{z}_{n+1}$ ). Here $z_{n}$ represents the computed solution at time $t_{n}$, and $h$ is the time step at time $t_{n}$. Expression (5) integrates over the time period $t_{n} \rightarrow t_{n+1}$. For the next time step $P_{n+1}$ is needed and the update from
$P_{n}$ into $P_{n+1}$ for the trapezoidal rule can be found as $P_{n+1}=-P_{n}+2 z_{n+1}\left(P_{n+1}=z_{n+1}\right.$ in case of backward Euler). Applying (5) to $i=C \dot{v}$ transforms the capacitor into a resistor $R_{n+1}$ in series with a voltage source $E_{n+1}$, with

$$
\begin{gathered}
R_{n+1}=\frac{h}{2 C} \\
E_{n+1}=\frac{h}{2 C} i_{n}+v_{n}
\end{gathered}
$$

valid at time instance $t_{n+1}$. In general, each dynamic circuit element can be transformed into a static network, valid at a certain time instance.

Now, consider a static network, consisting of PL components, resistors and (controlled) sources. Applying nodal analysis and taken the complementary variables $u, j$ of the $\mathrm{PL}$ components as the network variables, the complete behavior of the network can be given by a time indexed version of (3),

$$
\begin{aligned}
& j_{n+1}=M_{n+1} u_{n+1}+q_{n+1} \\
& j_{n+1} \geq 0, u_{n+1} \geq 0, j_{n+1}^{T} u_{n+1}=0
\end{aligned}
$$

Note that the second equation in (7) reflects a nonlinear operation and therefore this set of equations describes the complete large signal behavior of the nonlinear network at a certain time instance.

For class- $P$ networks, a technique does exist to obtain explicit expressions for the solutions of (7) [1], [2]. A short outline of this proof is as follows. Define the $[\cdot]$ operator as $\lfloor x\rfloor=\frac{1}{2}(|x|+x)$ and consider the scalar problem $j=d u+q$ valid under condition (2). For $q \geq 0$, the solution yields $j=q, u=0$ and for $q<0$ we obtain $j=0, u=-\frac{q}{d}$. Consequently, the solution may be written in an explicit formula, namely

$$
j=\lfloor q\rfloor, u=\left\lfloor-\frac{q}{d}\right\rfloor
$$

An interesting observation is that the discussed steps can completely be performed in a symbolic way [3]. Obviously, in symbolic expressions, it is not always clear if the LCP is of class- $P$, but this can be checked afterwards by filling in numerical values. The steps to analyze a PL dynamic network in a symbolic matter and/or to obtain the explicit formulas for the solutions of the network variables are the following:

- Apply a numerical integration method and transform dynamic elements into static replacement networks

- Reformulate the network equations in terms of the state vectors $u$, and $j$ of the PL models.

- Solve the LCP $j_{n}=D_{n} u_{n}+q_{n}, u_{n} \geq 0, j_{n} \geq 0$, $u_{n}^{T} j_{n}=0$ in an explicit way for time instance $t_{n} \rightarrow$ $t_{n+1}$. 
- Use the explicit formulas to express other network voltages and currents in terms of the network parameters.

Now formulas are obtained, describing the solutions of the nonlinear network explicitly in terms of time step $h$.

\section{EXAMPLES}

Consider the highly nonlinear PL network of Fig. 2 where the nonlinear resistor (4) (Fig. 1) is used. The network behavior can be described according to

$$
\begin{array}{cc}
C \frac{d v}{d t}=-\frac{v}{R_{2}}+\frac{e(t)-v}{R_{1}} & v \geq 0 \\
C \frac{d v}{d t}=\frac{e(t)-v}{R_{1}} & v<0
\end{array}
$$

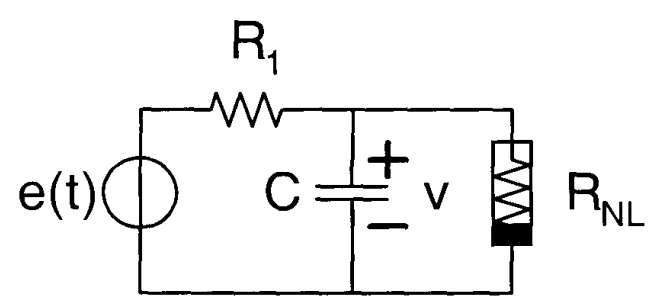

Fig.2. Nonlinear dynamic network

Let us apply a numerical integration method to transform the network into a static replacement network (Fig 3). This finally yields the following equations

$$
\begin{gathered}
e_{n+1}=\left[R_{1}+R_{2}+\frac{R_{1} R_{2}}{R_{n+1}}\right] j_{n+1}- \\
{\left[\frac{R_{1}}{R_{n+1}}+1\right] u_{n+1}-\frac{R_{1}}{R_{n+1}} E_{n+1}} \\
u_{n+1} \geq 0, j_{n+1} \geq 0, u_{n+1} \cdot j_{n+1}=0
\end{gathered}
$$

with $R_{n+1}=\frac{h}{2 C}$ and $E_{n+1}=\frac{h}{2 C} i_{n}+v_{n}$ (assuming trapezoidal rule). According to (8) the explicit formula for the solution of $(10)$ is given by

$$
\begin{aligned}
& j_{n+1}=\lfloor P\rfloor \cdot\left[R_{1}+R_{2}+\frac{R_{1} R_{2}}{R_{n+1}}\right]^{-1} \\
& u_{n+1}=\left\lfloor-P /\left(1+\frac{R_{1}}{R_{n+1}}\right)\right\rfloor
\end{aligned}
$$

with $P=e_{n+1}+\frac{R_{1}}{R_{n+1}} E_{n+1}$. The voltage $v$ across the capacitor can now be expressed as $v_{n+1}=R_{2} j_{n+1}$ $u_{n+1}$.

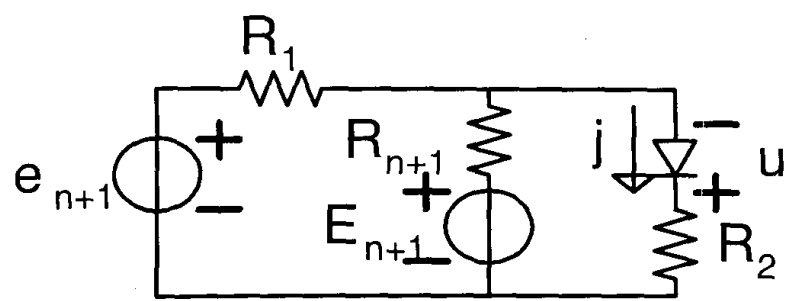

Fig. 3. Nonlinear static replacement circuit of Fig. 2 where the nonlinear resistor is modelled as in Fig. 1.

Note that (11) is highly nonlinear due to the special operator. Evaluating (11) leads to the nonlinear behavior of voltage across $C$ as shown in Fig. 4 .

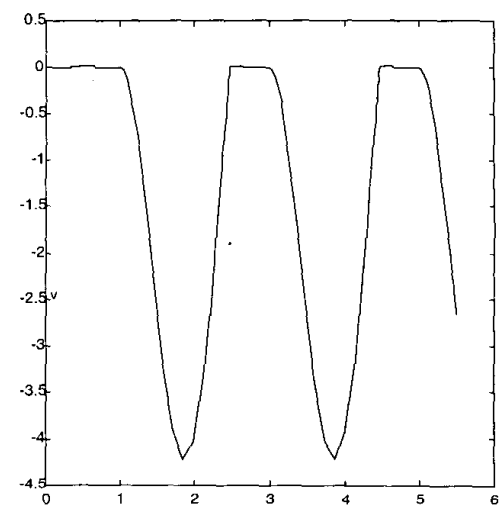

Fig. 4. Behavior of voltage $v$.

As second example consider the circuit in Fig. 5, taken from [4]. The network equations are given by

$$
\begin{gathered}
\dot{v}=\frac{1}{C} i \\
i=\frac{1}{L}\left(-v-R i+V_{r}\right)
\end{gathered}
$$

with the nonlinear resistor described by

$$
\begin{gathered}
V_{r}=\frac{1}{2} I_{r}+\left(\frac{1}{2}\right) u \\
j=-\frac{1}{2} I_{r}+\frac{1}{2} u \\
u \geq 0, j \geq 0, u^{T} j=0
\end{gathered}
$$

Instead of rewriting this network in terms of a LDCP and solve it accordingly [4], (5) is used to find a static LCP,

$$
j_{n+1}=\left(\frac{4+4 h+h^{2}}{8+6 h+h^{2}}\right) u_{n+1}+\frac{h P_{n 1}-2 P_{n 2}}{4+3 h+h^{2}}
$$


with $P_{n 1}, P_{n 2}$ the state parameters of the dynamic elements. The explicit formula for the solution of (15) is then readily obtained from (8),

$$
\begin{gathered}
j_{n+1}=\left\lfloor\frac{h P_{n 1}-2 P_{n 2}}{4+3 h+h^{2}}\right\rfloor \\
u_{n+1}=\left\lfloor-\frac{h P_{n 1}-2 P_{n 2}}{4+3 \bar{h}+h^{2}} \cdot \frac{8+6 h+h^{2}}{4+4 h+h^{2}}\right\rfloor
\end{gathered}
$$

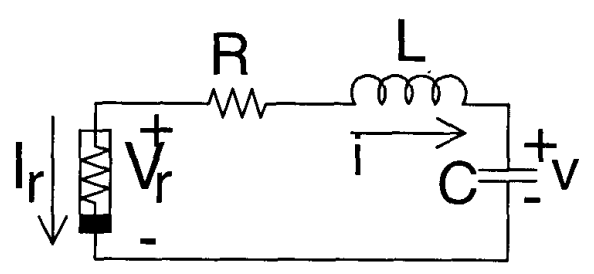

Fig. 5. Dynamic electrical network with one PL element [4].

Using the same numerical values as in [4] we have the initial condition set to $\left(P_{n 1}(0), P_{n 2}(0)\right)=$ $(-0.2,-0.2)^{T}$. Choosing the fixed step size $h$ equal to $h=0.001$ sec. transforms (15) into $j_{0}=0.5001 u_{0}+$ 0.0999 having the solution $u_{0}=0, j_{0}=0.0999$. At time instance $t_{n}=0.546+h$ we obtain $P_{n}=$ $(-0.2484,-0.0003)^{T}$ and $q_{n+1}=-2.0381 e^{-5}$. The LCP solution yields $j_{n+1}=\left|-2.0381 e^{-5}\right|=0$ and $u_{n+1}=\left\lfloor-2.0381 e^{-5} / 0.5001\right\rfloor=4.0751 e^{-5}$. The obtained results are exactly the same as in [4]. However, the proposed method does not need to detect changes in slope of the nonlinear resistor as in [4] because they are implicitly taken into account in the explicit formula.

\section{CLASS- $P$ PROPERTY}

The essential part within the proposed method is the class- $P$ property. One of the questions is of course what the impact is of the timestep on this property. Dynamic elements are replaced by resistive elements having positive values under any positive timestep value and will influence $M$. The additional sources in the replacement circuits of the dynamic elements will transfer to the vector $q$. If the circuit leaving out the dynamic elements is of class- $P$, then the circuit with dynamic elements will have this property under any time window. Changing timestep and/or integration rule will not change this fact. Class- $P$ property has a direct relation with the ideal diodes and thus with the static information of the network. This property is clear from the (simple) examples. Formula (10) has LCP matrix $M$ which is under all time windows positive, $M=\left[\frac{R_{1}}{R_{n+1}}+1\right] /\left[R_{1}+R_{2}+\frac{R_{1} R_{2}}{R_{n+1}}\right]$. The same holds for matrix $M$ in (16).

\section{CONCLUSION}

A method is presented to analyze large signal dynamic behavior of nonlinear networks. The nonlinear behavior is piecewise linear approximated and this allows to find the explicit formulas of the solutions in terms of the network voltages and currents. The dynamic elements are transformed into static replacement circuits having components values depending on the numerical integration method and time step. The expressions are described in terms of the nonlinear $\mid \cdot J$-operator, making the solutions valid over all PL segments. The method can be used to solve LDCPs too.

\section{REFERENCES}

[1] W.M.G. van Bokhoven, D.M.W. Leenaerts, 'Explicit formulas for the solutions of piecewise linear networks', IEEE Trans. Circ. Syst. part-I, sept. 1999

[2] D.M.W. Leenaerts, W.M.G. van Bokhoven, Piecewise Linear Modeling and Analysis, Kluwer Academic Pub., New York, 1998

[3] D.M.W. Leenaerts,"Symbolic analysis of large signals in nonlinear systems", proc. ISCAS, Orlando, pp. VI:5-8, 1999

[4] D.M.W. Leenaerts," On linear dynamic complementarity systems", IEEE Trans. Circ. Syst. part-I, pp.1022-1026, 1999

[5] W. Kruiskamp, D. Leenaerts, "Behavioral and macro modeling using piecewise linear techniques", Int. Journal on Analog Integrated Circuits and Signal Processing, 10, no. 1/2, pp.67-76, 1996

[6] J. Vlach, K. Singhal, Computer methods for circuit analysis and design Van Nostrand Reinhold, New York, 1983

[7] D.M.W. Leenaerts, "Further Extensions to Chua's Explicit Piecewise Linear Function Descriptions", Int. Journal of Circuit Theory and Appl., 24, pp.621-633,1996 